\title{
Comparison of genetic and drug resistance patterns in Pseudomonas aeruginosa isolated from the burn wound patients using primers 272 and 277
}

\author{
Z. Mahmmudi ${ }^{1}$ A. A. Gorzin*2 and A. Emami ${ }^{3}$ \\ ${ }^{I}$ Islamic Azad University, Kazeroon, Iran \\ ${ }^{2}$ School of Medicine, Shiraz University of Medical Sciences, Shiraz, Iran \\ ${ }^{3}$ Department of Microbiology, Shiraz Burn Research Center, Shiraz University of Medical Sciences, Shiraz, \\ Iran School of Medicine, Shiraz University of Medical Sciences, Shiraz, Iran
}

\begin{abstract}
Pseudomonas aeruginosa is one the main causes of opportunistic infections, especially in subjects with weakened immune systems such as patients with burns. Carbapenems such as imipenem (IMP) and meropenem (MEM) are among the most important antimicrobial drugs which are used to treat $P$. aeruginosa isolates resistant to penicillin and aminoglycosides. Given that identify the source of contamination and the resulting pattern of isolates resistant transmission in health centers play an important role in the control of hospital infections, the aim of this study was to determine antibiotic susceptibility and genetic patterns of $P$. aeruginosa bacteria isolated from patients admitted to Qutb al-Din Shirazi hospital. Of 200 samples collected from hospitalized burn patients at Qutb al-Din Shirazi Hospital Shiraz, Iran, 50 (25\%) isolates were confirmed as P. aeruginosa using standard biochemical tests. Antibiotic susceptibility of the isolates was examined through 11 antibiotics recommended by CLSI using the Kirby-Bauer method. RAPD-PCR technique with short primers of 272 and 277 were used to evaluate the genetic relationship among isolates. Of the antibiotics used, the most sensitive was found in gentamicin (50\%) and the highest resistances were observed in nalidixic acid (90\%), erythromycin (90\%), and cefotaxime (90\%). DNA fingerprinting was able to identify the genetic patterns of 18 and 15 in the form of bands 22 and 17 by RAPD-PCR technique with primers 272 and 277, respectively. RAPD-PCR is a technique for genotyping patterns with high resolution in epidemiological studies and bacterial polymorphism. The results of this study showed that antibiotic resistance in isolates of $P$. aeruginos $a$ is on the rise and there are different patterns in the hospital wards. The children's ward had just common genetic patterns with other wards of the hospital; this was the result of contamination in other parts of the hospital. Thus, according to the data of this study, there is a serious need to control the sources of infections by physicians and staff when they are working in several sectors in order to control and prevent the transmission of the bacteria.
\end{abstract}

KEY WORDS: CARBAPENEMS, P. AERUGINOSA, BIOCHEMICAL TESTS, GENETIC PATTERNS, BURN WOUNDS, RAPD-PCR TECHNIQUE

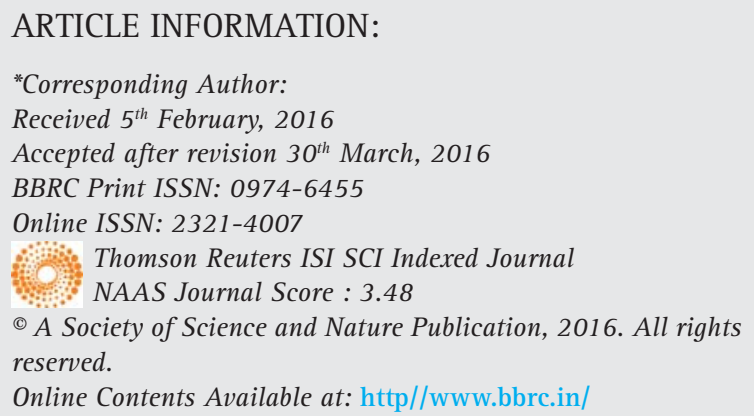




\section{INTRODUCTION}

One of the important problems in global health care is burn lesions, which puts patients at risk of hospital infections. Nosocomial infections are one of the most important medical problems worldwide, leading to outbreaks of infectious diseases in the community and are a major cause of morbidity and mortality, increased length of hospitalization, increased medical care cost and health problems. Hospital infections are created as limited or disseminated due to the pathogenic reactions associated with the infectious agents or their toxins in the hospital. The majority of nosocomial infections are caused by opportunistic agents such as $P$. aeruginosa. These bacteria have been identified as the most important bacteria that cause hospital infections. New methods of hospital cares have reduced the burn wound infections, but there is still the risk of deadly infections in severe burns, especially in developing countries and may result in the death of several thousand people annually.These cylindrical bacteria are mobile and classified accordance with the similarity of rRNA and common characteristics of culture. They smell like grape and grow easily on many media at 37 to $42{ }^{\circ} \mathrm{C}$. These oxidase positive microorganisms do not ferment carbohydrates and are regarded as the second most common gram-negative bacteria (21\%) in nosocomial infections (Trautner et al., 2004, Mermel, 2011).

These bacteria cause damages to the first line of defense and lead to dangerous infections in the skin of patients suffering from burns. The genetic patterns of $P$. aeruginosa by PCR-based molecular methods, compared to phenotypic methods are less affected by environmental factors, play a crucial role in tracking the routes of pathogen transmission. According to earlier studies, molecular techniques have higher ability to distinguish and repeatability than phenotypic tests and these progresses are due to their ability to distinguish small genomic and molecular stability, compared to phenotypic profiles of the same species, (Maki , 1994). In studies carried out in USA, for example, Shah et al., in a study in 2010 during six-month period found that of 125 bacterial samples, $90(72 \%)$ isolates were $P$. aeruginosa and the rest ones were $25 \mathrm{E}$. coli $(20 \%)$ and 10 staphylococcus (8\%), respectively (Shah et al., 2010). Meanwhile, a study was performed by Damron et al., in 2012. The most common causative organisms of burn wounds infections were respectively included Staphylococcus (48\%), Klebsiella (35\%) and P. aeruginosa (25\%) (Damron et al ., 2012).

Maki et al., in 1997 isolated and identified 85 isolates $P$. aeruginosa during a year from patients in ICU with routine bacteriological tests. Then their antibiotic susceptibility was determined with different disks; 50\%,
$70.5 \%$ and $17.6 \%$ of isolates were resistant to ceftazidime, cefotaxime and imipenem, respectively (Maki et al., 1997). Another earlier study was done by Pritt et al., in 2007, on blood, urine and fluids samples using MacConkey agar and blood agar media as well as antibiogram test according to NCCLS standards by Kirby Bauer method. During 1 year, 89 isolates of $P$. aeruginosa were isolated from samples and the most prevalence was observed in urine samples about 51\%. These organisms were resistant to ceftazidime and imipenem, respectively, 56\% and 24\% (Pritt et al., 2007).

Ekrame et al.,(2011) conducted at burn injury hospital of Ahwaz, Iran; 70 isolates suspected to P. aeruginosa were collected, of which 44 isolates were confirmed using biochemical tests, RAPD-PCR molecular techniques create reproducible electrophoretic patterns using short primers 272, and genetic fingerprinting using this technique showed 13 band patterns with the length of 180 to 2700 bp. Each isolates showed between 3 and 6 bands of these 13 patterns, which included 9 different genetic patterns (I - IX). The maximum frequency was related to genetic pattern I and the lowest frequency was observed in genetic patterns V and VIII, respectively, with 50\% and 20\% bacteria (Ekrame et al., 2011).

According to studies mentioned in many countries, resistance among strains of $P$. aeruginosa is increasing relative to beta-lactam compounds. For the treatment of infections caused by strains resistant to beta-lactam compounds is used carbapenems (imipenem-meropenem) that are resistant to beta-lactamases. Carbapenems are artificially obtained from Thienamycin antibiotic. Thienamycin, one of the most potent naturally produced antibiotics known thus far, was discovered in Streptomyces cattleya in 1976. Carbapenems such as imipenem and meropenem are the most important antimicrobial antibiotics which are used to treat resistant strains of $P$. aeruginosa. Imipenem inhibits the action of transpeptidation through strong adhesion to $\mathrm{PBP}_{1}$ and $\mathrm{PBP}_{2}$ and is resistant against many beta-lactamases as well as those that disable the third-generation cephalosporins. Imipenem well penetrates into gram-negative bacteria and is also effective against anaerobic bacteria (Dariouche, 2001).

The emergence of beta-lactamase enzymes has led to the phenomenon of resistance to antibiotics among many types of bacteria causing nosocomial infections and thus the treatment of infections is faced with serious problems. Beta-lactamases can hydrolyze wide range of Beta-lactams, including cephalosporins (third and fourth generations), penicillins and carbapenems (Maki et al., 1997). Today, there are four classes from A-D according to the molecular classification done based on nucleotide sequences and amino acids in the beta-lactamases. Betalactamase classes $\mathrm{A}, \mathrm{C}$ and $\mathrm{D}$ act through mechanisms based on serine while class B needs zinc for their activi- 
ties (Dariouche, 2001).Group A can hydrolyze penicillin and cephalosporin antibiotics that have been identified in bacteria such as Escherichia coli and Klebsiella pneumoniae (Trautner et al., 2004).Group B is able to hydrolyze carbapenem in bacteria such as Pseudomonas aeruginosa and Serratia marcescens (Trautner et al., 2004). Group C, generally, leads to the kind of chromosomal resistance and especially in Enterobacter (MacFaddin , 2000). Group D hydrolyze antibiotics such as oxacillin and cloxacillin with high strength (MacFaddin, 2000).

Since Pseudomonas aeruginosa is an important factor for nosocomial infection, especially in burn patients, so knowing how to release the special strains of bacteria has epidemiological importance to find the sources of infection, propagation mechanism and quick control, and because there is apparently particular genetic patterns of bacterial infections, the aim of this study was to evaluate the genetic patterns of resistant and susceptible strains of $P$. aeruginosa isolated from burn patients with RAPD - PCR technique to determine the diversity and genetic differences among patients admitted to a center as well as the origin will be identified.

\section{MATERIAL AND METHODS}

This experimental cross-sectional study was conducted over a period of one year (March 2013 to March 2014) in the burn research center of Shiraz University of Medical Sciences, Iran. 200 direct samples were collected by swab from the burn patients. It should be mentioned that all samples were obtained in the morning and before washing the patients and transported to the laboratory. The samples were cultured on blood agar and eosin methylene blue (EMB) and were microscopically and macroscopically evaluated for microbiological phenotypic.

Drug susceptibility testing: susceptibility of bacteria to different antibiotics was determined based on disk diffusion using the Kirby-Bauer method. Antibiotics were: chloramphenicol (CK), erythromycin (E), nalidixic acid (NA), meropenem (MEM), ceftazidime (CAZ), ciprofloxacin (CP), amikacin (AN), gentamicin (GN), imipenem (IMP), cefotaxime (CTX). Turbidity of medium was compared with $0.5 \mathrm{McF}$ arland.

Differential and biochemical tests for $P$. aeruginosa: the tests were performed as follows:

Test SIM: A colony of bacteria was inoculated by needle in sterile medium, production of $\mathrm{H} 2 \mathrm{~S}$, indole and motility of bacteria were characterized after adding Kovac's reagent.

Test TSI: a colony of bacteria was taken by sterile needle and cultured in depth and on the surface the TSI medium. Red color of the medium still remains due to lack of fermentation of glucose, lactose and sucrose on the surface and in depth.

Citrate test: A colony of bacteria was inoculated by needle in sterile medium. These bacteria due to the use of citrate as a carbon source change the color of medium from green to blue.

Growth at $42{ }^{\circ} \mathrm{C}$ : a colony of bacteria was cultured in two tubes containing Tryptic Soy Agar as sloping. For 12-24 hours, one of the tubes was placed at $35^{\circ} \mathrm{C}$ and the other at $42{ }^{\circ} \mathrm{C}$. $P$. aeruginosa grew in both temperatures.

Oxidase test: 1 to 3 drops of reagent oxidase was placed on a piece of paper inside a petri-dish. Culture was spread throughout the wet paper by platinum loop. In a positive reaction, $P$. aeruginosa bacteria immediately became dark purple, but oxidase negative organisms remain colorless.

Catalase test: Up to 2 drops of reagent catalase were poured onto a glass slide and some of the colonies were taken and solved in it. The positive reaction is characterized by the formation of oxygen bubbles, otherwise is considered as catalase-negative organisms.

\section{MOLECULAR METHODS}

To prepare buffer $\mathrm{X} 50$, tris base (242 g), glacial acetic acid $(57.1 \mathrm{ml})$, EDTA $0.5 \mathrm{M}(100 \mathrm{ml})$ were poured in a balloon and reached to a total volume of 1 lit by adding distilled water. The buffer was diluted 50 times using distilled water in order to provide a buffer X1 (for using in electrophoresis tank or gel). To prepare buffer X10, tris base ( $54 \mathrm{~g})$, Boric acid (27.5 g), EDTA 0.5 M (30 ml) were poured in a balloon and reached to a total volume of 0.5 lit by adding distilled water. The buffer was diluted 10 times using distilled water in order to provide a buffer X1 (for using in electrophoresis tank or gel).

Bacteria DNA extraction (Kit of CinnaGene-Iran): DNA was extracted from bacteria according to kit instructions, so that: First $100 \mu \mathrm{l}$ sterile phosphate buffered saline was poured into microtubes 1.5 and colonies were solved using a sterile needle. Then $400 \mu \mathrm{l}$ lysis buffers was added to each of the tubes and mixed. Next, $300 \mu \mathrm{l}$ precipitation buffer was added and gently mixed. The entire contents of microtubes were transferred to special filter pipes and centrifuged for one minute at 12000$13000 \mathrm{rpm}$. In the next phase, $400 \mu \mathrm{l}$ washing buffer (1) was added to filter pipes and centrifuged for one minute at 12000-13000 rpm again. Then $400 \mu$ l washing buffer (2) was added to filter pipes and centrifuged for one minute at 12000-13000 rpm again and this action 
was repeated once more. In the next phase, filter pipes was centrifuged without adding any substance at the same speed. In continuing, dry filters were removed and placed in sterile microtubes 1.5. In the final step, $50 \mu \mathrm{l}$ of dilution buffer, which had been already subjected inside thermoblock at $65^{\circ} \mathrm{C}$ for 3-5 min, was added to the filter pipes and centrifuged at the same speed. Upon completion of this step, filters were discarded and the contents of the tube containing the DNA of bacteria were stored at $-20^{\circ} \mathrm{C}$ for later testing.

DNA extraction by boiling: a loop-full isolates of $P$. aeruginosa from nutrient agar medium was taken and transferred to microtubes $1.5 \mathrm{ml}$ containing $500 \mu \mathrm{l}$ sterile distilled water and then the resulting solution was shake for 2 minutes by a shaker and placed in thermoblock for 20 min thermoblock at $75^{\circ} \mathrm{C}$. Then the supernatant containing bacterial DNA was transferred to a new microtube and stored at $-20^{\circ} \mathrm{C}$ for later testing.

\section{INVESTIGATION OF P. AERUGINOSA ISOLATES BY RAPD-PCR TECHNIQUE}

RAPD-PCR reaction as a quick method for DNA fingerprinting is the same normal PCR reaction, except that only short nucleotide primers are used in this method (10 nucleotides containing 50\% guanine and cytosine). In this way, various regions of the genome are simultaneously reproducible in PCR reactions (Christensen et al., 1985). Initial cycles are carried out at low temperatures of $37{ }^{\circ} \mathrm{C}$ to 50 ${ }^{\circ} \mathrm{C}$ for binding the primer to random locations within the genome, then the temperature is increased and the reaction continues for other 30-35 cycles; in fact, only more appropriate locations will be amplified among the initial random locations. If the template strand primer binding sites are at a convenient distance and have the proper orientation relative to each other, DNA polymerase is able to follow the distance between the two primers connected. With careful optimization can be achieved about 50 to 100 different DNA fragments, (Maki et al., 1997).

\section{IDENTIFYING THE OPTIMAL TEMPERATURE OF RAPD-PCR TECHNIQUE}

In this study, two primers (Bioneer Germany) (ten nucleotides) 272 and 277 were considered for RAPD-PCR reactions with the following sequences:

\section{5'-AGCGGGCCAA-3' \\ 5'-AGGAAGGTGC-3'}

First, master mix was prepared based on the compositions listed in the table above, which had not the genomic DNA isolates studied and $45 \mu \mathrm{l}$ of it was divided into microtubes $2.0 \mathrm{ml} .5 \mathrm{ml}$ of genomic DNA extracted ( $50 \mathrm{ng}$ ) was added to each microtube to reach the final volume of genomic amplification reaction to $50 \mu \mathrm{l}$. All the preparation steps of master mix and distribution were performed on ice. Negative controls were used to infer the probable contamination, which consisted of all components of genomic amplification without template DNA. And also a positive control was used included components of genomic amplification reaction with DNA of $P$. aeruginosa ATCC27853 as a representative of the pseudomonas complex. Following program to the number of 35 cycles were used for genomic amplification reaction. After electrophoresis and emerging bands, the right temperature for each of the three primers used in RAPD-PCR technique was defined as $42^{\circ} \mathrm{C}$ for primer 272 and $38^{\circ} \mathrm{C}$ for primer 277 .

Statistical analysis: The data was analyzed using software SPSS version sixteenth, Excell, (Microsoft office 2012), analysis of variance and T test $(\mathrm{p}<0.05)$.

Cluster analysis: clustering of results obtained from the electrophoresis of PCR products was carried out by software NTSYSpc (Numerical Taxonomy and Multivariate Analysis System). For each primer was drawn a dendrogram as well as an overall dendrogram derived from all the bands amplified by the primers. Finally matrix obtained from Jaccard similarity coefficients and UPGMA method (Unweighted Pair Group Method with Arithmetic Mean) was used to calculate the genetic distance.

\section{RESULTS AND DISCUSSION}

Of 200 burn patients, 117 (58\%) were males and 83 (42\%) were females; 83 (41\%), 54 (27\%), 35 (17\%) and 28 (14\%) samples were respectively related to men's ward, women's ward, children's ward and the ICU. Percentage of drug resistance in 50 isolates of $P$. aeruginosa has been shown in Figure 1.

All 50 isolates of $P$. aeruginosa DNA was extracted using kits, and was confirmed using 16srRNA gene. The genetic patterns of isolates were determined using RAPDPCR technique with two primers and the results were compared. Primer 272 in 18 genetic patterns of $P$. aeruginosa demonstrated altogether 86 bands and primer 277 in 15 genetic patterns of $P$. aeruginosa showed 65 bands. The numbers of polymorphism bands, which are based on the variation in the number of base pairs of bands, were 22 and 17 in the primers of 272 and 277, respectively. Performance of primers 272 and 277 were as follows: 45.3 and 34.2 , so the present study demonstrated higher performance of primer 272 than the other primer and results of this research were consistent with other studies on primer 272. Discriminatory power and detection of primer percentages were $43.1 \%$ and $33.4 \%$ in the primers 272 and 277 , respectively. In the present study it was found that discriminatory power and detection related to primer 272 


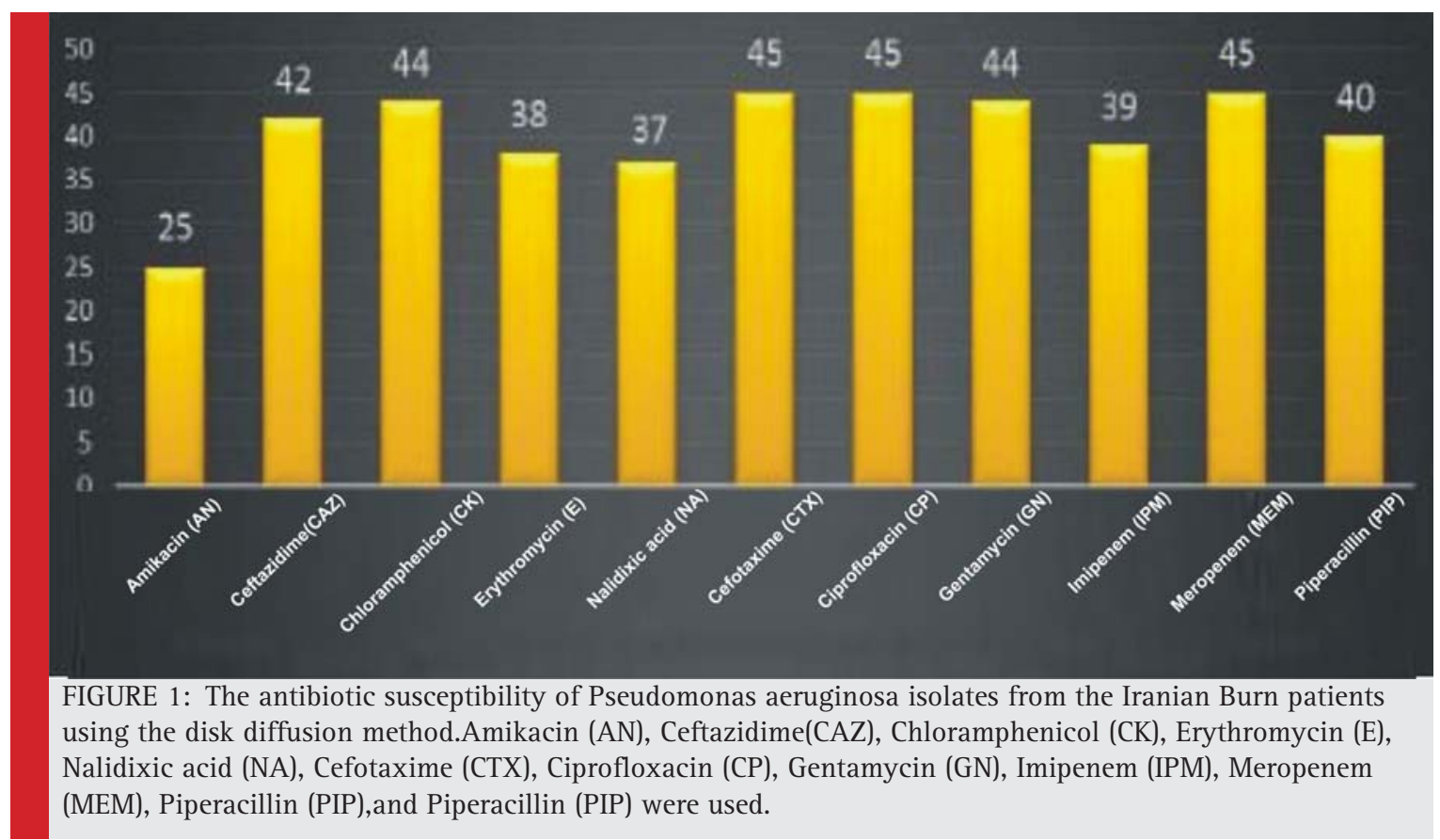

were more than the other primer and this confirms same thing we expected. Data analysis showed that over 95\% of carbapenem antibiotic resistance was observed in Pseudomonas aeruginosa strains isolated from patients hospitalized at burn ward of Qutb al-Din Shirazi hospital.

This experimental study was performed on 200 samples taken from patients by using standard microbiological tests to identify isolates of $P$. aeruginosa that 50 samples were identified as $P$. aeruginosa isolates. Antibiotic susceptibility of isolates was used in accordance with Kirby-Bauer disk diffusion method. Antibiotics discs included gentamicin, amikacin, piperacillin, nalidixic acid, erythromycin, ciprofloxacin, cefotaxime, meropenem, imipenem, ceftazidime and chloramphenicol that of 11 antibiotics used the most sensitivity was observed to gentamicin (25 samples, 50\%), and the least sensitivities (90\%) were associated with nalidixic acid, erythromycin and cefotaxime. But the rest of their sensitivities to antibiotic discs were as follows: 16\% imipenem; $12 \%$ meropenem; 24\% amikacin; 26\% chloramphenicol; 12\% piperacillin; 22\% ceftazidime; 20\% ciprofloxacin.

A study conducted by Costerton et al., in 2005, showed that $72 \%$ of the samples were infected by $P$. aeruginosa and in the next places were Escherichia coli, Staphylococcus aureus, Candida and Proteus, respectively (Costerton et al., 2005). The results of the study were not consistent and infection of Pseudomonas aeruginosa isolates was much more than this study, this could indicate a lack of hygiene and inappropriate sterilization and other factors in the study of Costerton. In investigation of
Maki et al (2006), the most common causative organism of burn wound infections were, respectively, staphylococcus, klebsiella and $P$. aeruginosa (Maki et al., 2006). The prevalence of $P$. aeruginosa isolates in this study (25\%) was similar to present work and it was shown that, unlike previous years, the prevalence of these bacteria in burns patients has been decreased.

The prevalence of the bacterium was $80 \%$ in a study conducted by Mahenthiralingam et al., in 2009 in the USA, actually more than our research (Mahenthiralingam et al., 2009). In a study by Pugashetti et al., (2008) in the Netherlands, the prevalence of bacteria involved in infections over a period of two years was as follows: Staphylococcus aureus (70\%), Pseudomonas aeruginosa (15\%), Klebsiella (9\%), Candida (5\%) and Proteus (1\%) (Pugashetti et al., 2008). The prevalence of $P$. aeruginosa isolates in this study was lower than ours, which represents the difference of bacteria involved in infections in various parts of the world. In a study, 89 cases of $P$. aeruginosa were isolated from different clinical specimens, which the highest prevalence was found in urine samples by $51.5 \%$ and were resistant to Ceftazidime (56\%) and imipenem (24.7\%), representing the annual increase (Akanji et al.,2011).

This study showed the increased resistances to imipenem and ceftazidime antibiotics are consistent with several studies conducted in the regions and neighboring countries, unlike industrialized countries; this indicates the importance of the proper use of these antibiotics in developed countries. 
A study was done by Hunter et al., in 2005 in Brazil. In this study, 140 isolates of $P$. aeruginos a were collected from clinical sources, the resistance patterns of strains to different antibiotic were as follows: 49.3\% gentamicin, 99.3\% cephalotin, 100\% thienamycin, 79.3\% ceftizoxime, 97.7\% cotrimoxazole, 35\% amikacin, 67.1\% carbenicillin, 65.7\% ceftriaxone, 58.6\% ciprofloxacin, 52.8\% piperacillin, 1.4\% imipenem, 65.7\% kanamycin, 72.1\% ofloxacin and 100\% ampicillin (Hunter et al., 2005). This study shows reduced antibiotic resistance in isolates of $P$. aeruginos a rather than present study, but concerning gentamicin was similar. Study of RAPD-PCR technique for typing isolates of $P$. aeruginosa using two ten-nucleotide primers created specific profiles and different genetic patterns were obtained with these primers, reflecting the ability of primers to differentiate the isolates (Govan et al., 2006).

A study was conducted to investigate polymorphism for $P$. aeruginosa isolated from cystic fibrosis patients through RAPD-PCR technique with primer 272 for genetic pattern of 58 by Maki et al., in 1997 in England; six different genetic patterns were obtained which showed high degree of polymorphism ((Maki et al., 1997). This research had the same results with this study .This study shows that the marker RAPD-PCR is a strong and effective technique in the identification and initial screening of samples to determine the differences among species, and identify at the level of strain as well as needs to spend lower time and cost compared with biochemical methods. The results showed that the profiles generated with the primers are comparable in terms of quality and repeatability. Sometimes, interpreting the results is difficult because of weak bands or their compactness, which may depend on the quality and quantity of DNA template.

Some points should be considered to ensure the power of amplification in RAPD-PCR techniques:

1. Samples should be evaluated by two different primers to confirm the differences among isolates;

2. All reactions should be repeated twice;

3. It is very important to standardize the PCR reaction mixture and unify the conditions for amplification;

4. Several dilution of DNA should be used for PCR reaction and the best dilution should be applied with gel analysis.

Now with regard to the importance of infection in burn patients and taking into account the high prevalence of $P$. aeruginosa isolates in the world and spread the bacteria resistance, quick identification and determination of therapeutic resistance pattern are very important. Today, carbapenems such as imipenem and meropenem are the main choice for treatment of infections caused by opportunistic gram-negative bacteria such as $P$. aeruginosa. According to the results obtained in this study and despite having more than 95\% of carbapenem antibiotic resist- ance in $P$. aeruginosa strains isolated from patients at Qutb al-Din Shirazi hospital, it seems that the efficacy of this drug have been greatly fallen and new patterns are needed to treatment. Known reasons for resistance of $P$. aeruginos $a$ isolates to carbapenem include: reduced the permeability of the outer membrane, increased expression of efflux pumps, changes in binding proteins and the presence of carbapenem hydrolyzing enzymes such as metallo beta lactamases. The data obtained from this study and other studies suggest that antibiotic resistance of $P$. aeruginosa to antibiotics is different in various parts of the world and even the resistances among species of pseudomonas are also different. According to the reasons presented, the incidence of resistance has often genetic origins and is due to add or remove the genetic fragments. This leads to changes in the pattern of chromosome sets in isolates of $P$. aeruginosa. As a result, the correct treatment of bacterial infections is needed to scrutiny of drug-resistant infectious agents and then prescribing the medications. Understanding the sensitivity and resistance status of these bacteria in hospitals is a necessity in order to determine the therapeutic plan in the initial encounter and control the resistance of bacteria to antibiotics. Today, various molecular techniques can be used to identify the genetic pattern of $P$. aeruginosa isolates.

According to studies, molecular techniques have higher power detection and repeatability of phenotypic tests and this progress is due to their ability to distinguish small genomic differences and high molecular stability compared to the phenotypic profiles of same strains. Advanced molecular typing methods such as RAPD-PCR are efficient, accurate and complete methods to identify genetic patterns of bacteria. This study also showed high polymorphism among isolates of P. aeruginosa related to Qutb al-Din Shirazi hospital. This was the first report of the genetic diversity associated with burn patients in the study area. According to the results, identify and classify the genetic patterns of $P$. aeruginosa bacteria isolated from burn patients are important in the pursuit of colonization the isolates in patients in terms of virulence severity and drug resistance and useful guide puts at the disposal of physicians in order to carry out preventative and treatment measures.

\section{REFERENCES}

Akanji BO, Ajele J0, Onasanya A. (2011). Genetic Fingerprinting of Pseudomona aeruginosa involved in nosocomial infection as revealed by RAPD-PCR markers. Biotech 10:70-77.

Christensen GD, Simpson WA, Younger JJ. (1985). Adherence of coagulase-negative Staphylococci to plastic tissue culture plates: a quantitative model for the adherence of Staphylococci to medical device. J Clin Microbiol 22:996-1006.

Costerton JW, Montanaro L, Arciola CR. (2005) Biofilm in implant infections: its production and regulation. Int J Artif Organs 28:1062-1068. 
Damron FH, Goldberg JB. (2012) Proteolytic regulation of alginate overproduction in Pseudomonas aeruginosa. Mol Microbiol 84:595-607.

Dariouche RO. (2001) Device-associated infections: a macroproblem that starts with micro- adherence. Clin Infect Dis 33:1567-1572. fibrosis. J Clin Microbiol 34:1129-1135.

Govan JRW. (2006) Mucoid strains of Pseudomonas aeruginosa: the influence of culture medium on the stability of mucus production. J Med Microbiol 8:513-522.

Hunter RP, Gaston AM. (2005). Numerical index of the discriminatory ability of typing systems: an application of Simpsons index of diversity. J Clin Microbiol 6: 2465-2466.

MacFaddin JF. (2000) Biochemical tests for the identification of medical bacteria. 3rd ed. [Lippincott Williams \&t Wilkins, Philadelphia, PA].

Mahenthiralingam E, Campbell ME, Foster J. (1996). Random amplified polymorphic DNA typing of Pseudomonas aeruginosa isolates recovered from patients with cystic

Maki DG. (1994). Infections caused by intravascular devices used for infusion therapy: pathogenesis, prevention, and management. In: Infections associated with indwelling medical devices. 2nd ed. Bisno AL and Waldovogel FA, editors.
American Society for Microbiology, Washington, DC. Pp 155212.

Maki, DG., Kluger DM, Crnich CJ. (2006) The risk of bloodstream infection in adults with different intravascular devices: a systematic review of 200 published prospective studies. Mayo Clin Proc 81:1159-1171.

Maki, DG, Weise, CE, Sarafın, HW. (1977). A semi quantitative culture method for identifying intravenous-catheter-related infection. N Engl J Med 296:1305-1309.

Mermel LA. (2011). What is the predominant source of intravascular catheter infections?.Clin Infect Dis 52:211-212.

Pritt B, O'Brien L, Winn W. (2007) Mucoid Pseudomonas in cystic fibrosis. Am J Clin Pathol 128:32-34.

Pugashetti BK, Metzger HM, Vadas L, (2008) Phenotypic differences among clinically isolated mucoid Pseudomonas aeruginosa strains. J. Clin. Microbiol. 16:686-691.

Shah H. Bosch W, Thompson KM, Hellinger WC. (2010) Intravascular catheter-related bloodstream infection. The Neuro Hospitalist. 3:144-151.

Trautner BW, Darouiche RO. (2004) Catheter-associated infections: Pathogenesis affects prevention. Arch Intern Med 164:842-850. 